\title{
TSPYL2 is an essential component of the REST/NRSF transcriptional complex for TGF $\beta$ signaling activation
}

\author{
MT Epping ${ }^{1,3}$, A Lunardi ${ }^{1,3}$, D Nachmani ${ }^{1}$, M Castillo-Martin ${ }^{2}$, TH Thin ${ }^{2}$, C Cordon-Cardo ${ }^{2}$ and PP Pandolfi, ${ }^{*, 1}$
}

REST/NRSF is a transcriptional repressor of neuronal genes that has been implicated in development and cancer. In epithelial tissues, REST acts as a tumor suppressor and in breast cancer, loss of REST is associated with disease recurrence and poor prognosis. Here, we identify TSPYL2 (also known as CDA1 and DENTT) as a novel component of the REST protein complex. We show that REST and TSPYL2 are regulators of TGF $\beta$ signaling and that cell-cycle arrest induced by TGF $\beta$ requires both REST and TSPYL2. Importantly, knockdown of REST or TSPYL2 resulted in transformation of human mammary epithelial cells. Mechanistically, we demonstrate that the TSPYL2/REST complex promotes TGF $\beta$ signaling by repressing the expression of genes, such as the proto-oncogene neurotrophic tyrosine kinase receptor $\mathrm{C}$ (TrkC). These data provide insight into the role of REST as a tumor suppressor in epithelial tissues through the regulation of the TGF $\beta$ pathway.

Cell Death and Differentiation (2015) 22, 1353-1362; doi:10.1038/cdd.2014.226; published online 23 January 2015

The transcription factor REST was originally discovered as a repressor of neuronal differentiation genes in non-neuronal lineages. Therefore, REST is considered as a master regulator of neurogenesis in development and maintenance of gene silencing. ${ }^{1-4}$ Recently, it has been recognized that aberrant REST function also has a role in human malignancies, largely depending on the cellular context. ${ }^{5}$ Focal genomic deletions targeting the REST locus have been found in colon adenocarcinoma and REST protein expression is often lost in small cell lung carcinoma cells. ${ }^{6,7}$ In breast cancer, the loss of full-length (FL) REST protein is associated with disease recurrence and poor prognosis. ${ }^{8}$ However, the underlying mechanism of how REST protects against transformation in epithelial tissues has remained largely elusive.

We have previously implicated the testis-specific protein, Y-encoded-like (TSPY-L) family of genes in cancer by identifying TSPYL5 expression as a prognostic marker of poor clinical outcome in breast cancer. ${ }^{9}$ TSPYL5 can interfere with p53-dependent cell-cycle arrest and oncogene-induced senescence triggering p53 ubiquitination and protein degradation. ${ }^{9}$ Besides TSPYL5, the TSPYL family of genes includes several other members (four coding genes and one pseudogene) whose functions are still unknown. All five proteins (TSPYL1, TSPYL2, TSPYL4, TSPYL5, and TSPYL6) are characterized by a predicted nucleosome assembly protein (NAP) domain, although functional evidence of NAP activity has not been reported of any of the TSPYL family members. TSPYL2, which shares only partial homology with TSPYL5, has been proposed as a candidate tumor suppressor in carcinoma cells. ${ }^{10}$ TSPYL2 is a negative regulator of proliferation, induces $\mathrm{p} 21^{\mathrm{CDKN} 1 \mathrm{~A}}$, and inhibits anchorageindependent growth; however, the mechanism through which TSPYL2 may function as a tumor suppressor is poorly understood. ${ }^{10-12}$

In this study, we identify TSPYL2 as a component of the REST/NRSF transcriptional repressor complex and describe an important role for this complex in the regulation of the TGF $\beta$ pathway.

\section{Results}

Nuclear TSPYL2 enhances TGF $\beta$ signaling. To investigate the physiological role of the TSPYL2 protein, we started to characterize TSPYL2 cellular localization and its expression levels in a panel of human cell lines. Overexpression of FLAG-TSPYL2 in lung (A549), prostate (PC3), and osteosarcoma (U2OS) human cell lines showed an exquisite nuclear localization of TSPYL2 (Supplementary Figure S1A). These data were further confirmed by the nuclear localization of endogenous TSPYL2 after nucleus/cytoplasm biochemical fractionation (Supplementary Figure S1B). Functionally, overexpression of wild-type TSPYL2 in A549 lung adenocarcinoma cells, which express low levels of endogenous TSPYL2 (Supplementary Figure S1C), inhibited cell proliferation and induced p21, as compared with GFP which was used as a control (Figures $1 \mathrm{a}$ and $\mathrm{b}, t=0$ ).

To explore how TSPYL2 could inhibit cell proliferation, we started by asking whether TSPYL2 could affect particular growth inhibitory pathways. Since TSPYL2 had been

\footnotetext{
${ }^{1}$ Cancer Research Institute, Beth Israel Deaconess Cancer Center, Department of Medicine and Pathology, Beth Israel Deaconess Medical Center, Harvard Medical School, Boston, MA, USA and ${ }^{2}$ Department of Pathology, Mount Sinai School of Medicine, New York, NY, USA

${ }^{*}$ Corresponding author: PP Pandolfi, Cancer Research Institute, Beth Israel Deaconess Cancer Center, Department of Medicine and Pathology, Beth Israel Deaconess Medical Center, Harvard Medical School, 330 Brookline Avenue NRB Suite 1038A, Boston, 02215 MA, USA. Tel: +1 617 667 3289; Fax: +1 617 667 3330; E-mail: ppandolf@bidmc.harvard.edu

${ }^{3}$ These authors contributed equally to this work.

Abbreviations: ChIP, chromatin immunoprecipitation; GFP, green fluorescent protein; HDAC, histone deacetylase; HMEC, human mammary epithelial cell; NPTXR, neuronal pentraxin receptor; NRSF, neuron-restrictive silencer factor; qRT-PCR, quantitative reverse-transcriptase PCR; RE1/NRSE, repressor element 1/neuron restrictive silencer element; REST, RE1-silencing transcription factor; RNAi, RNA interference; shRNA, short hairpin RNA; siRNA, small interfering RNA; TGF $\beta$, transforming growth factor $\beta$; TGF $\beta$ RII, TGF $\beta$ receptor type II; TrkC, neurotrophic tyrosine kinase receptor C; TSPYL, testis-specific protein, Y-encoded-like

Received 02.7.14; revised 02.11.14; accepted 19.11.14; Edited by G Melino; published online 23.1.15
} 


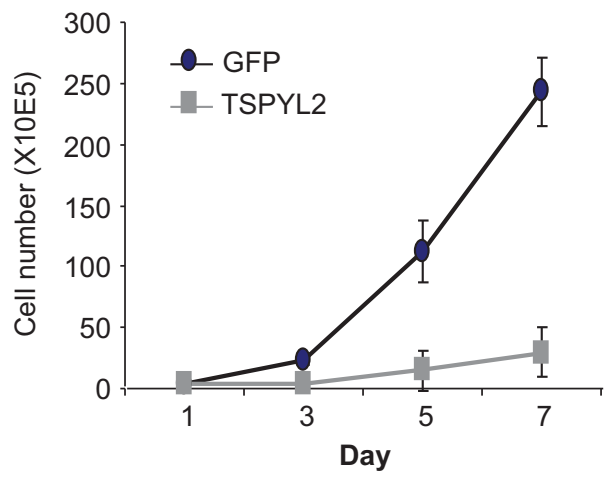

C
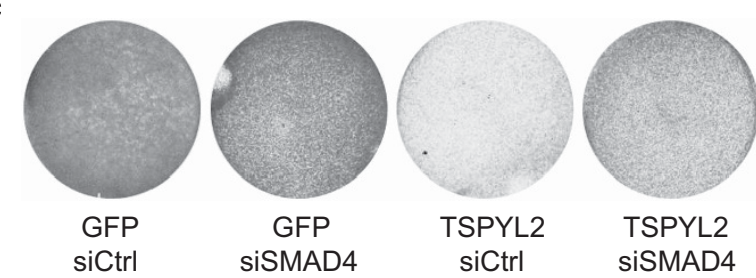

e

d

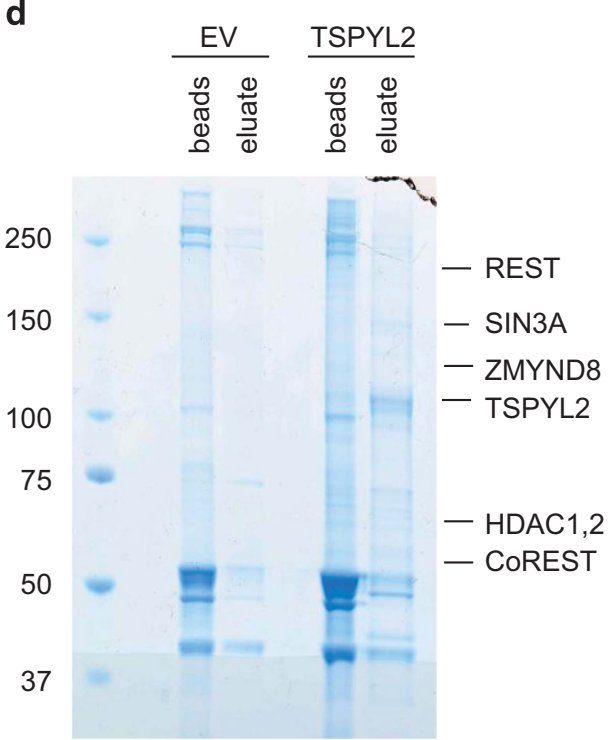

(i)

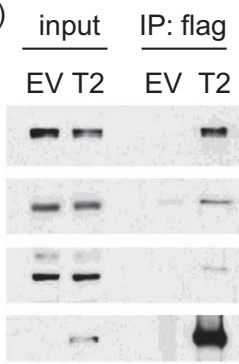

(ii)
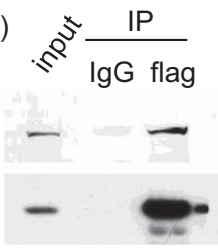

b

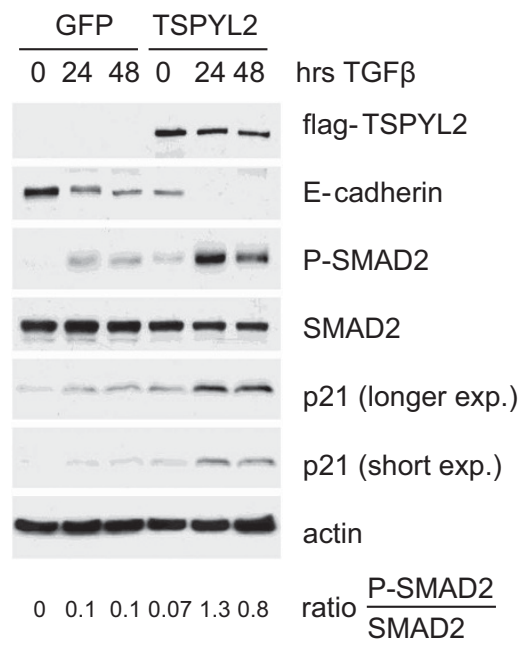

Figure 1 TSPYL2 is a positive regulator of TGF $\beta$ signaling and forms a protein complex with REST and corepressors. (a) Proliferation of A549 lung carcinoma cells upon infection with retroviruses expressing 3XFLAG-TSPYL2 or empty vector (EV). (b) A549 cells infected with TSPYL2 or GFP retroviruses were treated with TGF $\beta(5 \mathrm{ng} / \mathrm{ml}$ ) for 24 or $48 \mathrm{~h}$ and subjected to immunoblotting. TSPYL2 increased P-SMAD2 (S465/467) levels after TGF $\beta$ treatment and enhanced the effects of TGF $\beta$ on decrease of E-cadherin and induction of p21. Ratios between P-SMAD2 and SMAD2 were as indicated. (c) A549 cells were transfected with SMAD4 siRNA and subsequently infected with TSPYL2 or GFP retroviruses. Cell proliferation is shown by Coomassie staining 7 days after infection. (d) Coomassie-stained gel showing TSPYL2 and its associated proteins that were purified by co-immunoprecipitation (IP) using anti-flag antibody from nuclear extracts of A549 cells expressing 3XFLAG-TSPYL2. The immunoprecipitated proteins were eluted from the agarose beads and the eluates and post-elution beads were resolved by SDS-PAGE. A549 cells transduced with EV were used as a control. Mass spectrometric analysis revealed the identity of the proteins in the TSPYL2 eluate as indicated on the gel shown. (e) (i-ii) Co-IP using anti-flag antibody and western blotting of cells as in (a) showed the interaction between 3XFLAG-TSPYL2 (T2) and the indicated endogenous proteins. (iii-iv) Co-IP using antibodies against TSPYL2 and SIN3A showed the binding between endogenous TSPYL2, SIN3A, and REST proteins in nuclear extracts of HEK293 cells

associated with the pro-fibrotic role of TGF $\beta$ in diabetic atherosclerosis and diabetic retinopathy, ${ }^{13-15}$ we tested whether TSPYL2 could affect TGF $\beta$-driven phenotypes in our cancer cell systems. TGF $\beta$ is known to induce proliferation arrest in primary epithelial cells of various organs and the TGF $\beta$ pathway is frequently deregulated in malignancies. ${ }^{16,17}$ Phosphorylation and activation of SMAD2 (S465/467) and SMAD3 (S423/425) were increased in TSPYL2 
overexpressing cells, likely reflecting the activation of endogenous TGF $\beta$ signaling by TSPYL2 (Figure $1 \mathrm{~b}, t=0$; and Supplementary Figures S2A and B, $t=0$ ). Accordingly, TSPYL2 strongly enhanced SMAD activation in cells treated with TGF $\beta$ (Figure 1b; Supplementary Figures S2A and B) by increasing both the intensity and the duration of phosphoSMAD2 levels after prolonged (12-72 h) TGF $\beta$ treatment. This enhanced TGF $\beta$ response in turn drove a strong overexpression of $\mathrm{p} 21^{\mathrm{CDKN} 1 \mathrm{~A}}$ and downregulation of E-cadherin (Figure 1b). Moreover, TSPYL2 increased the basal transcript levels of the TGF $\beta$ target genes PAI1, JUNB, and SMAD7 and these were further increased upon treatment with TGF $\beta$ (Supplementary Figure S2C). To further assess the function of TSPYL2 in TGF $\beta$ pathway activation, we used SMAD4 siRNA and found that downregulation of SMAD4 impaired TSPYL2dependent changes in p21 and E-cadherin upon TGF $\beta$ treatment (Supplementary Figure S2D). Knockdown of SMAD4 also reversed the proliferation arrest imposed by TSPYL2, as SMAD4 siRNA rescued A549 cells from growth inhibition upon introduction of TSPYL2 (Figure 1c; Supplementary Figure S1D). Of note, TGF $\beta$ treatment did not affect the nuclear localization of TSPYL2 (Supplementary Figure S1B). Finally, the ability to hyperactivate TGF $\beta$ signaling was not shared by all TSPYL family members as TSPYL5 did not affect the TGF $\beta$ pathway (Supplementary Figure S2E).

TSPYL2 is a component of the REST repressor complex. Since TSPYL2 is a nuclear factor (Supplementary Figures S1A and B), its activity towards TGF $\beta$ might be related to a nuclear function. Accordingly, we tested the possible interaction of TSPYL2 with SMAD2/3 or SMAD4, but we did not observe any such associations (data not shown). Therefore, to gain insight into the mechanism and biological function of TSPYL2, we carried out flag-affinity purification of 3XFLAG-TSPYL2 from nuclear extracts of A549 cells to isolate TSPYL2-associated proteins. Mass spectrometric analysis identified many proteins in the TSPYL2-purified fraction that are components of the REST transcriptional repressor complex.1,2,4 The proteins associating with TSPYL2 were REST, SIN3A, HDAC1, HDAC2, CoREST, and ZMYND8 (Figure 1d; Supplementary Table S1). From the analysis it appeared that unique peptides corresponding to SIN3A were present in the highest abundance among the TSPYL2-associated proteins (Supplementary Table S1).

REST is a master regulator of neurogenesis and represses genes involved in neural differentiation in non-neuronal cells. ${ }^{1-4}$ Intriguingly, REST has been identified as a tumor suppressor in epithelial tissues, but the underlying mechanism has remained largely undiscovered. ${ }^{6}$ Gene silencing by REST depends on the recruitment of the co-repressors SIN3A and CoREST, which in turn recruit HDACs and ZMYND8 for neural-specific gene regulation. ${ }^{18-26}$ In addition to these proteins, our mass spectrometric analysis identified subunits of the SWI/SNF complex (BRG1, BAF170, and ISWI/SNF2H) and the nuclear remodeling NuRD complex (LSD1, MTA1, MTA2, RbAp46, and RbAp48) in the TSPYL2 purification, both known to be recruited by REST (Supplementary Table S1). To confirm our mass spectrometric results, we carried out coimmunoprecipitations in A549 and PC3 cell lines and found that endogenous SIN3A, HDACs, and REST could indeed co-precipitate with FLAG-TSPYL2 (Figure 1e, i-ii; Supplementary Figure S3A, i-ii). Consistently, we also observed interactions between FLAG-REST and endogenous TSPYL2, CoREST, and SIN3A in nuclear extracts of HEK293 cells, which express high endogenous levels of TSPYL2 (Supplementary Figure S3A, iii). Furthermore, we showed that the binding between TSPYL2, REST, SIN3A, and CoREST was constitutive and was not affected by TGF $\beta$ (Supplementary Figures S3B and C). Importantly, we assessed whether physiological binding between endogenous TSPYL2, SIN3A, and REST occurred in cells and observed associations between these endogenous proteins in HEK293 nuclear extracts (Figure 1e, iii-iv). Because of the association between TSPYL2 and the proteins in the REST silencing complex, we tested whether TSPYL2 could affect transcription using a heterologous reporter system. A fusion protein consisting of TSPYL2 and the DNA-binding domain of Gal4 (Gal4-TSPYL2) readily inhibited transcription of a Gal4-responsive luciferase reporter (Supplementary Figure S3D). Similarly, a Gal4-SIN3A protein was able to repress transcription of the reporter, while Gal4-TSPYL5 did not affect luciferase reporter activity (Supplementary Figure S3D).

Hence, nuclear localization, mass spectrometric analysis, co-immunoprecipitation experiments, and Gal4 reporter activity identified TSPYL2 as an important component of the REST multi-subunit transcriptional repressor complex.

TSPYL2 N-terminus domain mediates REST complex binding and TSPYL2 biological activity. To determine which domain of TSPYL2 was primarily involved in its biological function, we generated a series of 3XFLAGTSPYL2 deletion mutants (Figure 2a). The deletion mutants were subsequently introduced into A549 cells to allow comparisons with FL TSPYL2. Expression of TSPYL2 mutants 4 and 6 , as well as FL TSPYL2 triggered a proliferation arrest in A549 cells (Figure 2b). Next, we compared the three largest deletion mutants (numbered 4-6) to FL-TSPYL2 for binding to SIN3A by co-immunoprecipitation. The TSPYL2 mutants 4 and 6 , but not 5 , interacted with SIN3A similar to FL-TSPYL2 (Figure 2c). When we tested these mutants for their abilities to hyperactivate TGF $\beta$ signaling, we found that mutants 4 and 6 , however not 5, prolonged the elevation of phospho-SMAD2 levels up to $48 \mathrm{~h}$ after TGF $\beta$ treatment, when phospho-SMAD2 signal was already reduced to the steadystate level in control cells (Figure 2d). It followed from these data that the $\mathrm{N}$-terminus domain of TSPYL2, which is missing in mutant 5 , is the likely mediator of the biological function of TSPYL2. However, the fact that mutant 1 did not show the ability to arrest the cell cycle of A549 cells nor to bind SIN3A in co-immunoprecipitation experiments (Supplementary Figure S4A) was initially puzzling. A possible explanation for this apparent discrepancy might be the incorrect folding of the TSPYL2\#1 fragment when isolated from the rest of the protein. To test this possibility, we cloned TSPYL2\#1 downstream of the Gal4 DNA binding domain. Indeed, a Gal4TSPYL2\#1 (containing TSPYL2 domain 1, representing the $\mathrm{N}$-terminus) fusion protein did interact with SIN3A (Figure 2e), and, consistently, repressed transcription of a 


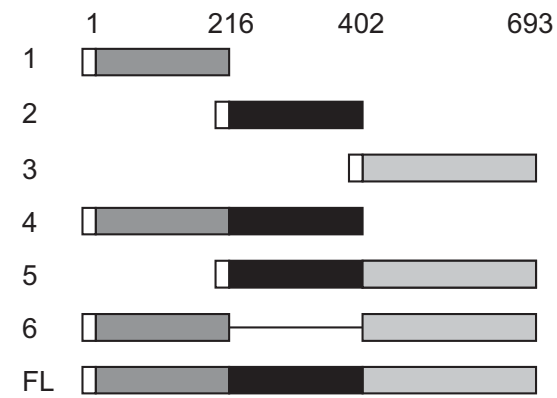

c

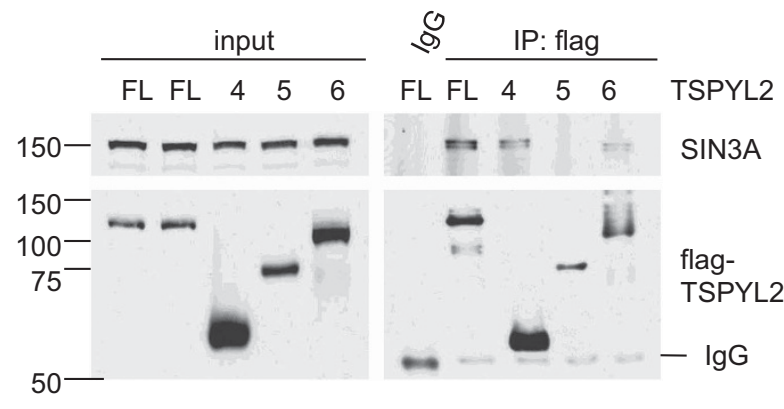

e

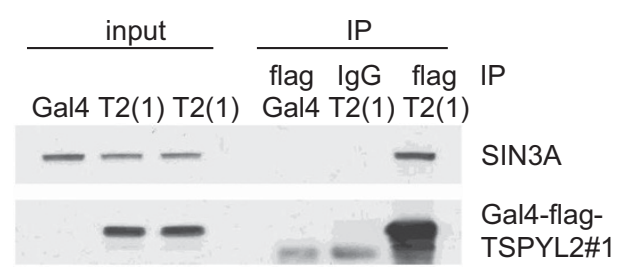

b

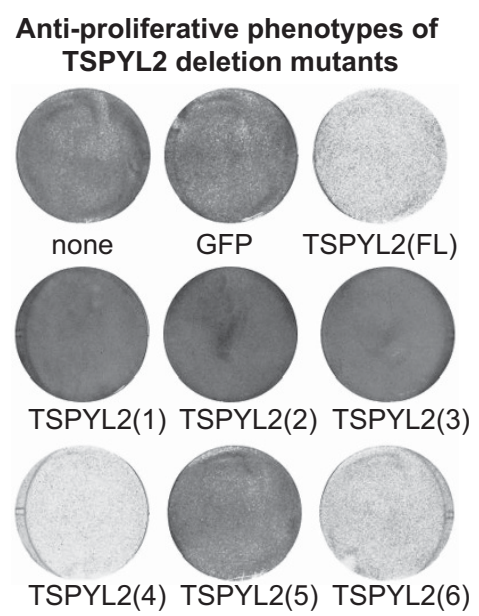

d $\frac{-}{-+} \frac{\mathrm{FL}}{-+} \frac{\# 4}{-++} \frac{\# 5}{-+}$

TSPYL2 TGF $\beta$ P-SMAD2

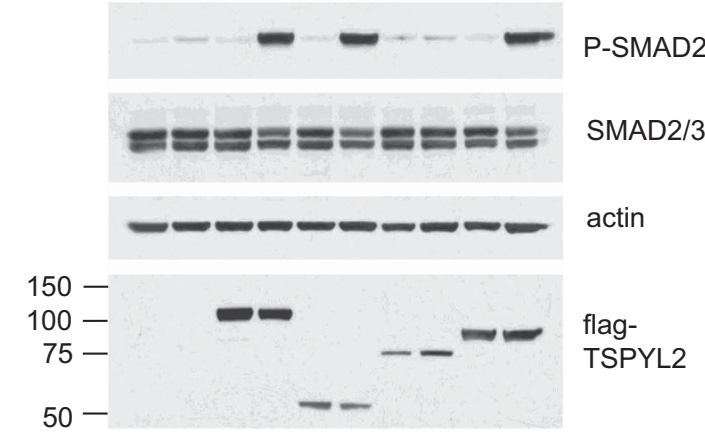

f shGFP shTSPYL2

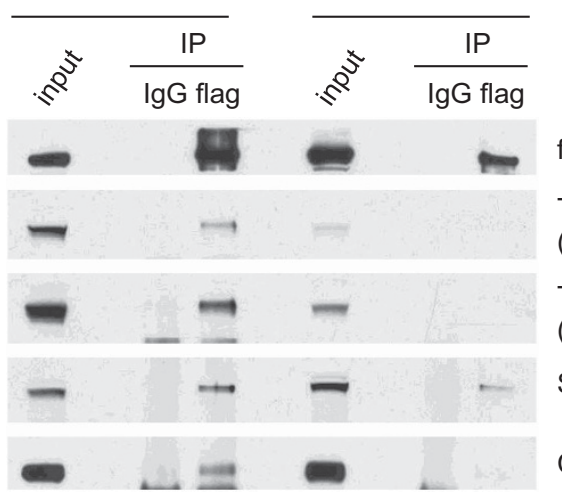

flag-REST

TSPYL2 (short exp.)

TSPYL2 (long exp.)

SIN3A

CoREST

Figure 2 TSPYL2 interacts with SIN3A and REST. (a) Schematic representation of the TSPYL2 deletion mutants that were generated and numbered 1-6. FL, full-length. The deletion mutants had N-terminal 3XFLAG tags and the positions of amino acids were as indicated. (b) A549 cells were infected with retroviruses encoding either FL 3XflagTSPYL2 or TSPYL2 deletion mutants 1-6 and the cells in the culture dishes were stained with Coomassie Blue after 7 days. (c) Co-immunoprecipitation showed the interactions between TSPYL2 deletion mutants 4-6 and endogenous SIN3A. (d) A549 cells were infected with retroviruses encoding the TSPYL2 deletion mutants 4-6 and treated with TGF $\beta$ $(5 \mathrm{ng} / \mathrm{ml})$ for $48 \mathrm{~h}$, followed by immunoblotting to assess the effects of the mutants on TGF $\beta$ signaling. (e) The binding between the N-terminus of TSPYL2 (domain 1) and endogenous SIN3A was demonstrated in HEK293 cells transfected with a plasmid encoding a Gal4-flag-TSPYL2 (domain 1) fusion protein (indicated as T2(1)), while a Gal4-flag protein (indicated as Gal4) served as a control. Immunoprecipitation was done using either an anti-flag antibody or control lgG. (f) HEK293 cells were co-transfected with FL REST and shRNA targeting TSPYL2 or GFP and after $72 \mathrm{~h}$, co-immunoprecipitation showed that the interaction between REST and CoREST and SIN3A was compromised upon knockdown of TSPYL2

Gal4-responsive reporter (Supplementary Figure S4B). Therefore, we concluded that the N-terminus of TSPYL2 is required for its biological function and for binding to the REST/SIN3A repressor complex.
To further analyze the role of TSPYL2 as one of the corepressors of REST, we tested where on the REST protein the interaction domain with TSPYL2 was located. REST contains two distinct repressor domains, one located in the 
$\mathrm{N}$-terminus that binds SIN3A and the other located in the C-terminus that interacts with CoREST. ${ }^{4}$ Co-immunoprecipitation experiments with REST deletion mutants showed that endogenous TSPYL2 associated with REST (322-1097) rather than the N-terminus of REST (1-323) in nuclear extracts (Supplementary Figures S4C and D). We then tested whether TSPYL2 interacted with the central domain of REST (322-841) or with its C-terminus (841-1097), and found that TSPYL2 was bound to the central domain of REST (322-841) (Supplementary Figure S5E). Importantly, the binding between REST, CoREST, and SIN3A was compromised after introduction of TSPYL2 shRNA (Figure 2f).

Loss of TSPYL2 or REST affects TGF $\beta$-dependent cellcycle arrest. Overall, the data suggested a functional connection between REST and the TGF $\beta$ pathway through TSPYL2. To this end, we infected human HaCaT cells, which express TSPYL2 and are sensitive to TGF $\beta$, with shRNA retroviruses to generate specific knockdown of TSPYL2, REST, and SMAD4 proteins (Figure 3a). HaCaT cells expressing control GFP shRNA were readily growth arrested by TGF $\beta$, but strikingly, cells with TSPYL2 or REST shRNA continued to proliferate upon addition of TGF $\beta$ (Figure 3b). Hence, cells with knockdown of either TSPYL2 or REST became insensitive to the cell-cycle arrest induced by TGF $\beta$. HaCaT cells with SMAD4 knockdown were used as controls and were completely resistant to TGF $\beta$ (Figure $3 b$ ). As the results represent the cumulative effects of TGF $\beta$ on cell proliferation and survival, we therefore cannot exclude that a fraction of the cells was lost due to cell death (Figure 3b). Insensitivity to TGF $\beta$ following downregulation of TSPYL2 or REST was also evident from gene expression analysis of PAl1, p21 ${ }^{\mathrm{CDKN} 1 \mathrm{~A}}$ and $\mathrm{p} 15^{\mathrm{CDKN} 2 \mathrm{~B}}$, which are involved in the proliferation arrest imposed by TGF $\beta{ }^{27}$ Transcript levels of these genes were diminished in HaCaT cells with TSPYL2, REST, or SMAD4 shRNA, concomitant with the observed resistance of the cells to TGF $\beta$ (Figure 3c). Next, we tested the consequence of knockdown of TSPYL2 and REST in transactivation assays using a $\mathrm{CAGA}_{12}$-luciferase reporter containing multimerized SMAD binding elements from the PAl1 promoter. ${ }^{28}$ TGF $\beta$ activated the reporter gene, but knockdown of either TSPYL2 or REST abrogated the activation of the reporter by TGF $\beta$ (Figure 3d; Supplementary Figure S4F). Overall, these data indicated that loss of TSPYL2 or REST resulted in cellular resistance to TGF $\beta$-dependent proliferation arrest.

Transcriptional repression of TrkC by the TSPYL2/REST complex enhances TGF $\beta$ oncosuppressive activity. REST and its associated corepressor complexes silence neuronal genes in non-neuronal lineages. ${ }^{1-4}$ Among the genes restricted by REST is the neurotrophic tyrosine kinase receptor $\mathrm{C}$ (TrkC). ${ }^{29}$ While Trk receptors are required for the development, function, and survival of the mammalian nervous system, aberrant expression of Trk receptors has been associated with oncogenic phenotypes in non-neuronal cells. The proto-oncogene TrkC allows transformation of mammary epithelial cells, while cells expressing TrkB acquire cell survival and metastatic capabilities. ${ }^{29,30}$ Of relevance, TrkC is also involved in the $t(12 ; 15)(p 13 ; q 25)$ translocation that has been detected in secretory breast cancer, congenital fibrosarcoma, and acute myeloid leukemia. ${ }^{31-34}$ This translocation results in the ETV6-NTRK3 (Tel-TrkC) chimeric oncoprotein, which is an initiating event in secretory breast carcinoma, a rare subtype of infiltrating ductal breast carcinoma originating from committed mammary progenitor cells. $^{31,32}$ Importantly, both TrkC and ETV6-NTRK3 have been reported to bind to and inhibit TGF $\beta$ RII, thereby negatively regulating SMAD phosphorylation and downstream TGF $\beta$ signaling. ${ }^{35,36}$

To investigate whether TSPYL2 is required for RESTmediated TrkC repression, we first overexpressed TSPYL2 in A549 cells and found that TSPYL2 decreased TrkC mRNA and protein levels (Figures $4 a$ and $b$ ). Interestingly, we also noticed that TrkC was induced by TGF $\beta$ in A549 cells, but that TSPYL2 completely abrogated this increase (Figure $4 a$ and $b$ ). An increase in TrkB has been observed previously in neurons that were exposed to TGF $\beta .^{37}$ Like TrkB, TrkC may itself be a TGF $\beta$ responsive gene, which would create a negative feedback loop between TGF $\beta$ and TrkC. Conversely, REST or TSPYL2 shRNA de-repressed the TrkC locus and allowed for increased TrkC expression levels (Figure 4c). Similar results were obtained in HEK293T cells (Supplementary Figure S5A). Next, we determined whether TGF $\beta$ signaling was directly affected by TrkC levels. Indeed, knockdown of TrkC resulted in elevated phospho-SMAD2 levels upon treatment with TGF $\beta$ (Figure $4 \mathrm{~d}$; Supplementary Figure $\mathrm{S} 5 \mathrm{~B})$. Consistently, we found that in co-immunoprecipitations, endogenous TrkC physically interacted with endogenous TGF $\beta$ RII (Figure 4e; Supplementary Figure S5C).

REST silences neuronal genes through directly occupying a conserved DNA sequence, known as RE1 or NRSE (repressor element $1 /$ neuron restrictive silencer element) ${ }^{1,2}$ that is present in its target genes. We therefore tested whether the $\mathrm{RE} 1$ sites in the genes encoding TrkC and neuronal pentraxin receptor (NPTXR) were occupied by REST and TSPYL2. Due to the unavailability of a ChIP-grade TSPYL2 antibody, we overexpressed HA-TSPYL2 in A549 cells to perform chromatin immunoprecipitation (ChIP) experiments. ChIP revealed enrichment of REST, TSPYL2, and SIN3A at proximal DNA regions encompassing the RE1 sites in the TrkC and NPTXR genes (Figure 4f; Supplementary Figure S5D). These data indicated that the REST/TSPYL2 complex is present on chromatin to inhibit the expression of neuronal genes, including TrkC.

REST was previously identified in a genome-wide RNAi screen as a tumor suppressor gene in human mammary epithelial cells (HMECs). ${ }^{6}$ Knockdown of REST triggers anchorage-independent proliferation of HMECs, which represents an in vitro hallmark of transformation. ${ }^{6}$ To determine whether TSPYL2 would be tumor suppressive in the same context, we tested whether loss of TSPYL2 would result in transformation in this assay. Strikingly, we found that HMECs infected with TSPYL2 shRNA retrovirus formed colonies in soft agar, similar to cells with REST shRNA (Figure $4 \mathrm{~g}$ ). Hence, downregulation of either REST or TSPYL2 was sufficient to cause transformation, consistent with the notion that both genes could act as tumor suppressors. HMECs display substantial constitutive TGF $\beta$ signaling in standard culturing condition while abrogation of this pathway causes 
a

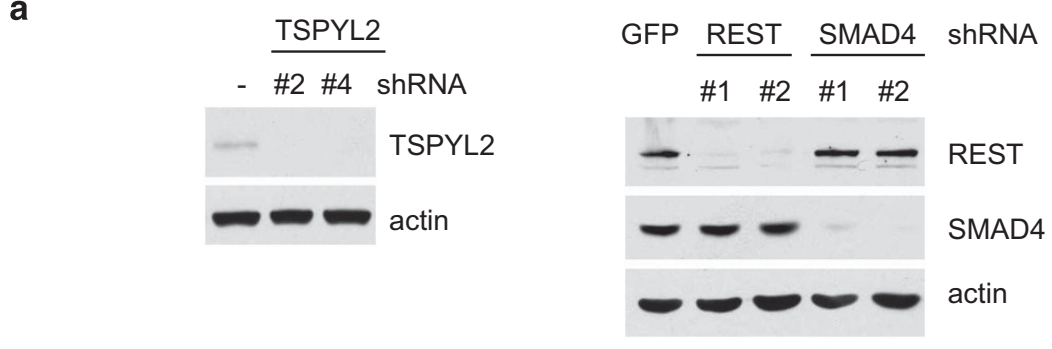

b
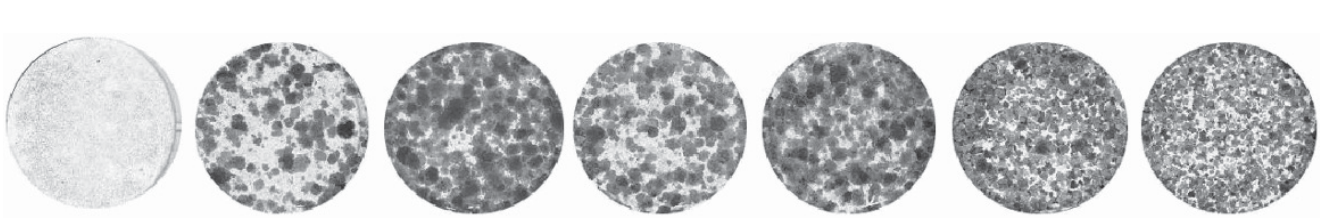

TGF $\beta$

$5 \mathrm{ng} / \mathrm{mL}$

14days

shRNA: GFP

TSPYL2 \#2

TSPYL2 \#4

REST \#1

REST \#2

SMAD4 \#1

SMAD4 \#2

C

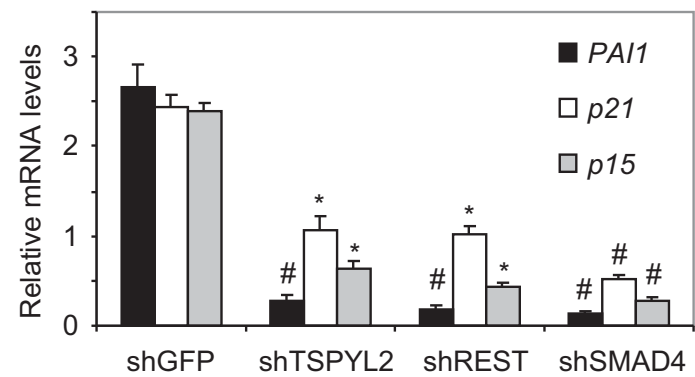

TGF $\beta$

$5 \mathrm{ng} / \mathrm{mL}$

14days

d
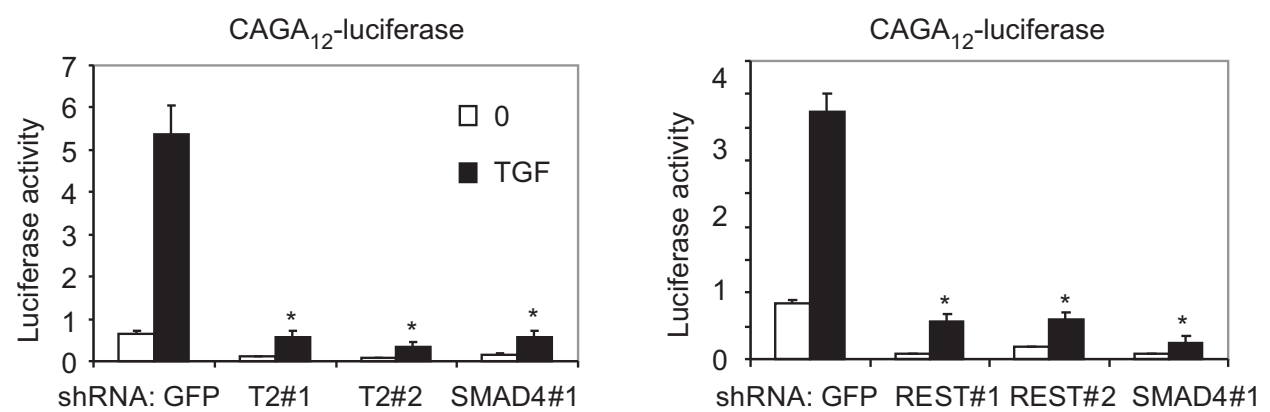

Figure 3 Loss of REST or TSPYL2 confers resistance to TGF $\beta$-induced proliferation arrest. (a) Immunoblotting confirming knockdown of TSPYL2, REST, and SMAD4 in the $\mathrm{HaCaT}$ cells transduced with specific shRNAs. (b) HaCaT cells were transduced with shRNA retroviruses targeting TSPYL2, REST, SMAD4, or GFP and subsequently cultured in TGF $\beta$ $(5 \mathrm{ng} / \mathrm{ml})$. Culture dishes were stained with Coomassie and photographed after 2 weeks. (c) Gene expression levels of three TGF $\beta$ target genes were detected by quantitative RT-PCR in the cells in (b). Relative mRNA levels of PAl1, p21, and p15 were normalized to the internal control GAPDH (mean+S.D., $n=4,{ }^{*} P<0.05,{ }^{\#} P<0.01$ ). (d) U2OS cells were co-transfected with a CAGA 12 -luciferase reporter in the presence of the indicated shRNAs and treated with TGF $\beta(5 \mathrm{ng} / \mathrm{ml})$ for $24 \mathrm{~h}$ before detection of luciferase activity (mean+S.D., $n=3)$

transformation. ${ }^{6}$ The transcript levels of PAl1 and SMAD7 were decreased in HMECs after knockdown of TSPYL2 or REST, likely reflecting the inhibition of endogenous TGF $\beta$ signaling in these cells (Supplementary Figure S6A). Moreover, introduction of a wild-type TrkC allowed colony formation in transformation assays and reduced PAl1 and SMAD7 mRNA levels in HMECs (Figure 4g; Supplementary Figure S6B).

Overall, these data indicate that TrkC is a key target of the TSPYL2/REST complex for the regulation of the tumor suppressive activity of the TGF $\beta$ pathway.

\section{Discussion}

Taken together, our findings provide novel insights into the function of TSPYL2, which have allowed us to reach several relevant conclusions.

First, we have identified TSPYL2 as an essential component of the REST protein complex that represses transcription of proneuronal genes in non-neuronal tissues. Our protein purification strategy demonstrated that TSPYL2 associates with many of the known cofactors for REST-mediated gene silencing.

Second, we have found that expression of TSPYL2 in epithelial cells is anti-proliferative by activating and 
a

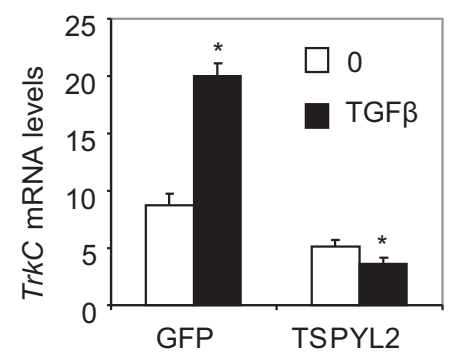

b

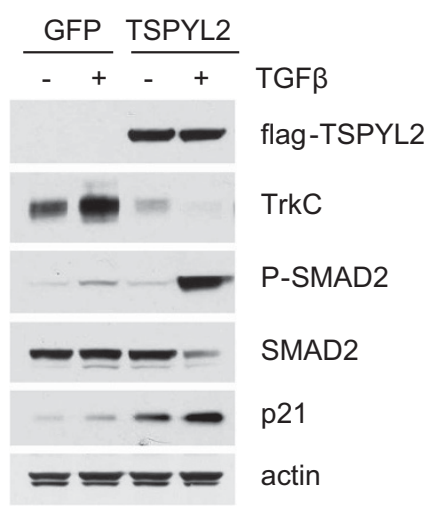

C

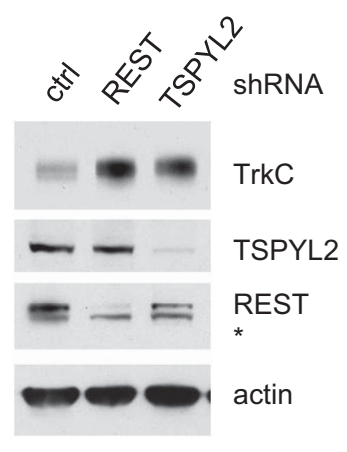

d

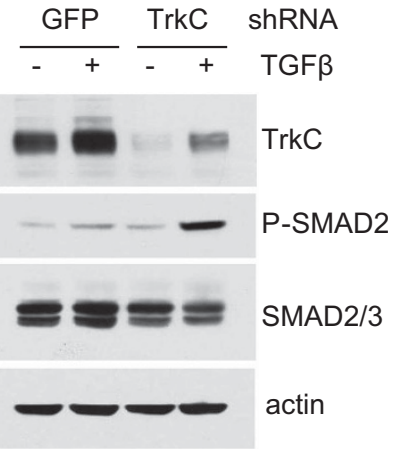

e

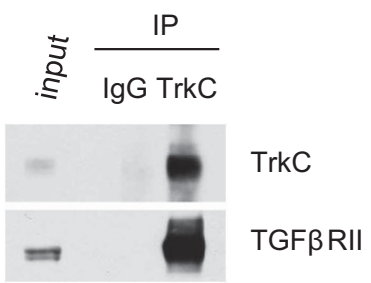

f
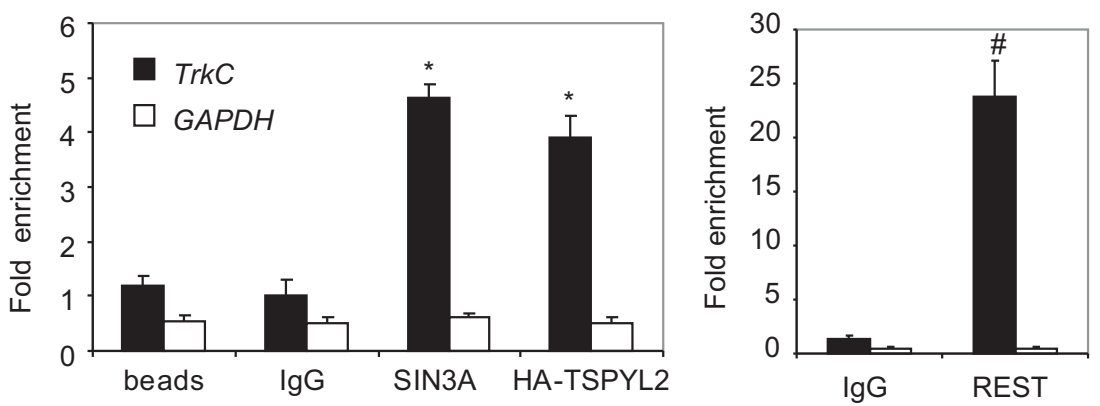

g

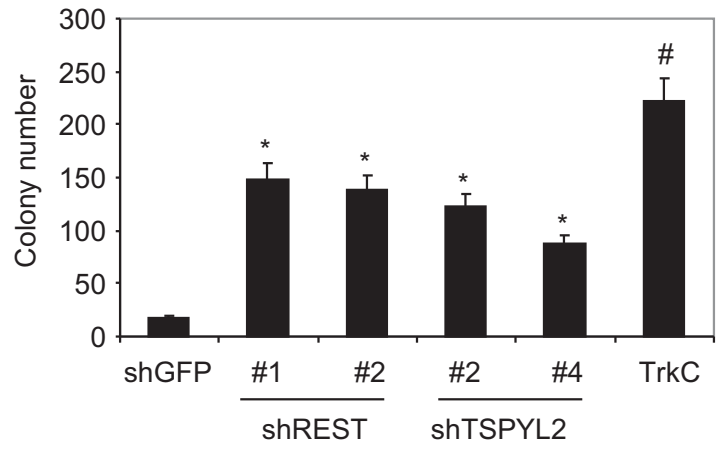

Figure 4 REST and TSPYL2 enhance TGF $\beta$ signaling through inhibition of TrkC expression. (a) Quantitative RT-PCR showed TrkC gene expression levels in A549 cells overexpressing TSPYL2 or GFP after treatment with TGF $\beta$ for $24 \mathrm{~h}$. Relative TrkC mRNA levels were normalized to the internal control GAPDH (mean+S.D., $n=4,{ }^{*} P<0.05,{ }^{\sharp} P<0.01$ ). (b) A549 cells were infected with TSPYL2 or GFP retroviruses, treated with TGF $\beta(5 \mathrm{ng} / \mathrm{ml})$ for $48 \mathrm{~h}$ and subjected to immunoblotting to show TrkC repression, and TGF $\beta$ pathway activation by TSPYL2. (c) Knockdown of REST or TSPYL2 de-repressed the TrkC locus resulting in increased TrkC levels (* indicates a non-specific band). (d) Immunoblot showing cells treated with TGF $\beta$ for $48 \mathrm{~h}$ after infection with TrkC shRNA. (e) Interaction between endogenous TrkC and TGF $\beta$ RIl proteins was shown by co-immunoprecipitation using anti-TrkC antibody. (f) ChIP followed by quantitative PCR was used to analyze the occupancy of TrkC RE1 site-proximal chromatin by REST, HA-TSPYL2, and SIN3A in A549 cells expressing HA-TSPYL2. GAPDH promoter was used as a control (mean+S.D., $n=3$ ). (g) Anchorage-independent proliferation of HMECs infected with TSPYL2 or REST shRNA retroviruses was tested in soft agar assays. Expression of full-length TrkC cDNA in HMECs was also tested for cellular transformation. Colony numbers were as depicted (mean $+S$.D., $n=3$ ) 
potentiating TGF $\beta$ signaling. The TSPYL2/REST complex is required for proper responses to TGF $\beta$, as downregulation of TSPYL2 or REST results in resistance to TGF $\beta$-induced proliferation arrest as well as to inhibition of TGF $\beta$ target gene expression.

Mechanistically, we propose a model in which the multi-subunit complex consisting of REST, TSPYL2, and the associated corepressors is required for full transcriptional repression of RE1containing neuronal genes, thereby modulating the sensitivity of cells to the anti-proliferative effect of TGF $\beta$, one of the most important barriers to transformation in epithelial cells (Figure 5). Among the genes silenced by the REST/TSPYL2 complex we found TrkC, a neurotrophic receptor already described as a potent inhibitor of TGF $\beta$ signaling, although we cannot exclude that additional genes may be involved.

TGF $\beta$ is known to inhibit cellular proliferation in normal epithelial development, for example, in ductal and glandular mammary tissue. ${ }^{38}$ During the initial phases of breast carcinogenesis, TGF $\beta$ signaling acts as a powerful tumor suppressor through the transcriptional induction of genes such as p15 ${ }^{\mathrm{INK} 4 \mathrm{~B}}, \mathrm{p} 16^{\mathrm{INK} 4 \mathrm{~A}}, \mathrm{p} 19^{\mathrm{ARF}}, \mathrm{p} 21^{\mathrm{CIP} 1}, \mathrm{PAI}$, and p57 and the consequent inhibition of tumor growth. ${ }^{39}$ However, epithelial cancer cells progressively acquire resistance to the cytostatic effects driven by TGF $\beta$, and the tumor progresses towards advanced stages. ${ }^{39}$

Loss of REST protein, or the expression of aberrant REST transcript variants encoding truncated REST proteins, has been reported in several types of human malignancies of epithelial origin, including breast, lung, and colon cancers. ${ }^{6-8}$ In breast cancer, the loss of FL REST protein, in favor of a shorter carboxyterminal truncated form has been described in a small fraction of cases and is associated with disease recurrence and poor prognosis. ${ }^{8}$ Accordingly, we provide evidence that both TSPYL2 and REST are important for tumor suppression in epithelial cells, as knockdown of either gene or overexpression of TrkC was sufficient for the acquirement of the ability of anchorageindependent growth in HMECs. Interestingly, high TrkC expression was recently observed in comparison with normal breast tissue in about $84 \%$ of the infiltrating ductal carcinomas analyzed..$^{40}$ Additionally, through in vitro and in vivo experimental studies, the authors demonstrated that overexpression of FL wild-type TrkC is sufficient to promote proliferation and colony formation, as well as tumor growth in mouse models of breast cancer. Mechanistically, they attributed the oncogenic activity of TrkC to its ability to activate MAPK and PI3K pathways. ${ }^{40}$ Intriguingly, REST has also been linked to PI3K signaling, albeit in a different context. ${ }^{6}$

Together, our results unveil an important tumor suppressive function of the TSPYL2/REST complex in epithelial cancers through gene silencing and the transcriptional maintenance and potentiation of the tumor suppressive TGF $\beta$ pathway.

\section{Materials and Methods}

Reagents and plasmids. TGF $\beta$ was obtained from Peprotech (Rocky Hill, NJ, USA) and used at a final concentration of $5 \mathrm{ng} / \mathrm{ml}$ unless indicated otherwise. SB431542 was purchased from Ascent Scientific/Abcam Biochemicals (Cambridge, $\mathrm{MA}, \mathrm{USA}$ ) and used at $20 \mu \mathrm{M}$. The pBabePuro-3Xflag-Nbio retroviral vector (EV, empty vector) has been described previously. ${ }^{9}$ Human TSPYL2 cDNA was cloned into the aforementioned vector to generate pBabePuro-3Xflag-Nbio-TSPYL2, as well as in pBabePuro after addition of an N-terminal HA tag, resulting in pBabePuro-HATSPYL2. TSPYL2 deletion mutants were generated by PCR amplification of the indicated protein domains, which were subsequently ligated into the EV vector. Human REST cDNA was cloned into the pCR3-CMV-3Xflag plasmid, which resulted in the 3Xflag-REST expression vector. The 3Xflag-REST (1-323) and (322-1097) constructs were created by digestion of REST CDNA at an internal Nde1 restriction site. The 3Xflag-REST (322-841) and (841-1097) constructs were generated by PCR amplification. To generate pCR3-CMV-Gal4-flag, a cDNA encoding the Gal4 DNAbinding domain (1-147) was cloned upstream of the flag tag in pCR3-CMV-flag. TSPYL2 FL and deletion mutants were inserted downstream of the Gal4-flag to generate the Gal4-flag-TSPYL2 constructs. Similarly, Gal4-flag-Sin3A and Gal4-flagTSPYL 5 were made by cloning Sin3A and TSPYL5 cDNAs into PCR3-CMV-Gal4-flag. The pBabePuro-3Xflag-Nbio-TSPYL5 vector has been described before. ${ }^{9}$ Human TrkC cDNA was cloned into pBabePuro to express TrkC. SMAD4 and TrkC siRNA pools were obtained from Dharmacon (Lafayette, CO, USA). shRNA constructs were generated in the pRetroSuper plasmid and target sequences are listed in Supplementary Table S2.

Cells and retroviral infections. A549, U2OS, HaCaT, HEK293, and Phoenix cells were cultured in DMEM supplemented with $10 \%$ FBS, L-glutamine, and antibiotics. HMECs expressing hTERT and SV40 large T-antigen (TLM-HMECs) ${ }^{6}$ were cultured in mammary epithelial growth medium (MEGM, Lonza, Walkersville, MD, USA). Retroviruses were generated by transfection of Phoenix packaging cells and retroviral supernatants were used for infection after addition of $4 \mu \mathrm{g} / \mathrm{ml}$ polybrene.

Protein purification and mass spectrometry. Nuclear extracts of A549 cells were prepared by incubation of the cells in hypotonic lysis buffer $(10 \mathrm{mM}$ Hepes pH7.9, $0.2 \mathrm{mM}$ EDTA, $1 \mathrm{mM}$ DTT) for $10 \mathrm{~min}$, addition of Triton X-100 to a final concentration of $0.1 \%$ and centrifugation to collect the cell nuclei. Nuclei were lysed in lysis buffer $(50 \mathrm{mM}$ Tris $\mathrm{pH} 7.5,10 \%$ glycerol, $150 \mathrm{mM} \mathrm{NaCl}, 0.2 \% \mathrm{NP}-40$, protease inhibitor cocktail EDTA-free (Complete, Roche, Indianapolis, IN, USA), $10 \mathrm{mM} \mathrm{NaF}$, $0.2 \mathrm{mM} \mathrm{Na}_{3} \mathrm{VO}_{4}, 2.5 \mathrm{mM}$ sodium pyrophosphate, $2.5 \mathrm{mM}$ sodium $\beta$-glycerophosphate, $1 \mathrm{mM}$ DTT, $1 \mathrm{mM}$ PMSF) for $30 \mathrm{~min}$, sonicated, and centrifuged, before incubation with anti-FLAG M2 affinity gel (Sigma, St. Louis, MO, USA). The immunoprecipitates were washed with wash buffer $(50 \mathrm{mM}$ Tris $\mathrm{pH} 7.5,150 \mathrm{mM} \mathrm{NaCl}, 0.1 \% \mathrm{NP}-40$, protease inhibitor cocktail EDTA-free (Complete, Roche), $10 \mathrm{mM} \mathrm{NaF}, 0.2 \mathrm{mM}$ $\mathrm{Na}_{3} \mathrm{VO}_{4}, 2.5 \mathrm{mM}$ sodium pyrophosphate, $2.5 \mathrm{mM}$ sodium $\beta$-glycerophosphate, $1 \mathrm{mM}$ DTT, $1 \mathrm{mM}$ PMSF) and eluted using $200 \mathrm{ng} / \mu \mathrm{l}$ 3XFLAG peptide (Sigma) in wash buffer. The eluates were added to Laemmli sample buffer and separated on Bis-Tris gradient gels (NuPAGE Novex, Invitrogen, Grand Island, NY, USA). The gels were stained with SimplyBlue Safe Stain (Invitrogen) and excised bands were subjected to in-gel trypsinization followed by LC-MS/MS analysis.

Western blotting and immunoprecipitation. For western blotting, cells were lysed in Ripa buffer (50 mM Tris pH 8.0, $150 \mathrm{mM} \mathrm{NaCl}, 1 \% \mathrm{NP}-40,0.5 \%$ deoxycholic acid, and $0.1 \%$ SDS) supplemented with protease inhibitors (Complete,

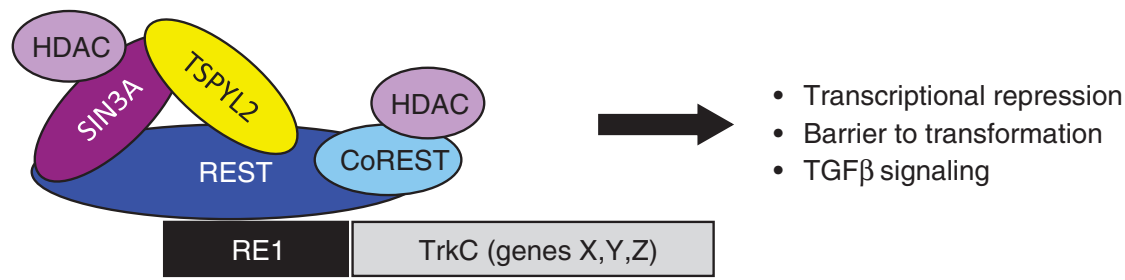

Figure 5 Model depicting the REST repressor complex with TSPYL2. The intact complex is required for transcriptional repression of genes, such as TrkC, thereby regulating $\mathrm{TGF} \beta$ signaling and functioning as a barrier against transformation of epithelial cells 
Roche), briefly sonicated, and resolved on SDS-PAGE gels. The proteins were transferred onto nitrocellulose membranes and detected by chemiluminescence after incubation with the indicated antibodies. For co-immunoprecipitation, cells were lysed as described above for protein purification and the lysates were incubated with the indicated antibodies and protein $A / G$ agarose to retrieve coimmunoprecipitated protein complexes, which were subsequently subjected to western blotting. Antibodies for western blotting and co-immunoprecipitation were as follows: the TSPYL2 antibody was from Proteintech Group (Chicago, IL, USA) and the REST antibody (07-579) was from Upstate/Millipore (Bedford, MA, USA). Antibodies against P-SMAD2 (S465/467), P-SMAD3 (S423/425), E-cadherin, TrkC, and PARP were from Cell Signaling (Danvers, MA, USA). Flag (M2), flag (rabbit), and actin antibodies were from Sigma. SMAD2/3 antibodies were obtained from Millipore and the SMAD2 antibody was from BD Transduction Laboratories (Franklin Lakes, NJ, USA). SMAD4 (B-8), Sin3A (AK-11), HDAC1 (H-51), HDAC2 (H-54), HA $(\mathrm{Y}-11), \mathrm{HSP} 90(\mathrm{H}-114)$, and $\mathrm{p} 21^{\mathrm{ClP}}(\mathrm{F}-5)$ were from Santa Cruz Biotechnology (Dallas, TX, USA). TGF $\beta$ RII antibodies were from Cell Signaling and Upstate, and the CoREST antibody was from Bethyl Laboratories (Montgomery, TX, USA).

Luciferase reporter assay. U2OS cells were co-transfected with $\mathrm{CAGA}_{12}$ luciferase and CMV-renilla internal control plasmids and the indicated shRNA constructs using the calcium phosphate precipitation method. TGF $\beta$ was added $96 \mathrm{~h}$ after transfection and cells were cultured for another $24 \mathrm{~h}$ before lysis. Relative luciferase activity was measured using the Dual-Luciferase Reporter system (Promega, Madison, WI, USA) in an automated 96-well plate reader (Promega) and normalized to renilla internal control values. Similarly, U2OS cells were cotransfected with a luciferase reporter containing $5 X$ Gal4 response elements (Gal4TK-luciferase), CMV-renilla, and the indicated Gal4-TSPYL2 plasmids before detection of luciferase activity $72 \mathrm{~h}$ after transfection.

qRT-PCR. Total RNA was extracted using Trizol reagent (Invitrogen) and used to prepare first-strand cDNA using the SuperScriptlll polymerase (Invitrogen) or iScript reverse transcriptase mix (Bio-Rad, Hercules, CA, USA). Quantitative real-time PCRs were done using SYBR Green master mix (Roche or Qiagen, Germantown, $M D, ~ U S A$ ) in a thermal light cycler (Roche). Relative mRNA levels of each gene shown were normalized to the expression of GAPDH. qRT-PCR primers are listed in Supplementary Table S3.

Chromatin immunoprecipitation. ChIP assays were performed according to the Upstate/Millipore protocol with small modifications. A549 cells expressing HATSPYL2 were crosslinked with $1 \%$ formaldehyde for $10 \mathrm{~min}$ at room temperature. Nuclear extracts were prepared in hypotonic buffer as described above and nuclei were lysed in nuclear lysis buffer ( $50 \mathrm{mM}$ Tris pH8.0, $10 \mathrm{mM}$ EDTA, $1 \%$ SDS, protease inhibitors) and sonicated. The chromatin was diluted 10-fold in IP buffer (0.01\% SDS, 1.1\% Triton X-100, $1.2 \mathrm{mM}$ EDTA, $16.7 \mathrm{mM}$ Tris pH8.0, $167 \mathrm{mM} \mathrm{NaCl}$, protease inhibitors, $1 \mathrm{mg} / \mathrm{ml} \mathrm{BSA}$ ) and incubated with $5 \mu \mathrm{g}$ of specific antibodies overnight at $4^{\circ} \mathrm{C}$. The antibodies used for ChIP were Sin3A (K-20) and HA (Y-11) antibodies from Santa Cruz Biotechnology and REST antibody (07-579) from Upstate/Millipore. Immunocomplexes were collected on protein A/G-agarose beads preadsorbed with sonicated single-strand DNA. Following five sequential washes, DNA was eluted using $1 \% \mathrm{SDS}$ in $0.1 \mathrm{M} \mathrm{NaHCO}_{3}$ and cross-linked complexes were reversed in $0.2 \mathrm{M} \mathrm{NaCl}$ by heating at $65^{\circ} \mathrm{C}$ for $4 \mathrm{~h}$. The DNA in the immunoprecipitates was purified and resuspended in $30 \mu \mathrm{l}$ TE. The DNA sequences were detected by quantitative real-time PCR using sets of primers designed to amplify the RE1-containing regions of the TrkC and NPTXR genes or the GAPDH promoter (Supplementary Table S3).

\section{Conflict of Interest}

The authors declare no conflict of interest.

Acknowledgements. The help and advice of all members of the Pandolfi laboratory is gratefully acknowledged. We thank Thomas Garvey for insightful editing This work was supported by the MMHCC/NCI grant U01 CA 141496-04 to PPP. AL has been supported in part by a fellowship from the Istituto Toscano Tumori (ITT, Italy).

1. Chong JA, Tapia-Ramirez J, Kim S, Toledo-Aral JJ, Zheng Y, Boutros MC et al. REST: a mammalian silencer protein that restricts sodium channel gene expression to neurons. Cell 1995; 80: 949-957.

2. Schoenherr CJ, Anderson DJ. The neuron-restrictive silencer factor (NRSF): a coordinate repressor of multiple neuron-specific genes. Science 1995; 267: 1360-1363.
3. Ballas N, Grunseich C, Lu DD, Speh JC, Mandel G. REST and its corepressors mediate plasticity of neuronal gene chromatin throughout neurogenesis. Cell 2005; 121: 645-657.

4. Ballas N, Mandel G. The many faces of REST oversee epigenetic programming of neuronal genes. Curr Opin Neurobiol 2005; 15: 500-506.

5. Majumder S. REST in good times and bad: roles in tumor suppressor and oncogenic activities. Cell Cycle 2006; 5: 1929-1935.

6. Westbrook TF, Martin ES, Schlabach MR, Leng Y, Liang AC, Feng B et al. A genetic screen for candidate tumor suppressors identifies REST. Cell 2005; 121: 837-848.

7. Kreisler A, Strissel PL, Strick R, Neumann SB, Schumacher U, Becker CM. Regulation of the NRSF/REST gene by methylation and CREB affects the cellular phenotype of small-cell lung cancer. Oncogene 2010; 29: 5828-5838.

8. Wagoner MP, Gunsalus KT, Schoenike B, Richardson AL, Friedl A, Roopra A. The transcription factor REST is lost in aggressive breast cancer. PLoS Genet 2010; 6: e1000979.

9. Epping MT, Meijer LA, Krijgsman O, Bos JL, Pandolfi PP, Bernards R. TSPYL5 suppresses p53 levels and function by physical interaction with USP7. Nat Cell Biol 2011; 13: 102-108.

10. Kandalaft LE, Zudaire E, Portal-Nunez S, Cuttitta F, Jakowlew SB. Differentially expressed nucleolar transforming growth factor-beta1 target (DENTT) exhibits an inhibitory role on tumorigenesis. Carcinogenesis 2008; 29: 1282-1289.

11. Chai Z, Sarcevic B, Mawson A, Toh BH. SET-related cell division autoantigen-1 (CDA1) arrests cell growth. J Biol Chem2001; 276: 33665-33674.

12. Tu $\mathrm{Y}, \mathrm{Wu}$ W, Wu T, Cao Z, Wilkins $\mathrm{R}$, Toh BH et al. Antiproliferative autoantigen CDA1 transcriptionally up-regulates p21(Waf1/Cip1) by activating p53 and MEK/ERK1/2 MAPK pathways. J Biol Chem2007; 282: 11722-11731.

13. Pham Y, Tu Y, Wu T, Allen TJ, Calkin AC, Watson AM et al. Cell division autoantigen 1 plays a profibrotic role by modulating downstream signalling of TGF-beta in a murine diabetic model of atherosclerosis. Diabetologia 2010; 53: 170-179.

14. Tu Y, Wu T, Dai A, Pham Y, Chew P, de Haan JB et al. Cell division autoantigen 1 enhances signaling and the profibrotic effects of transforming growth factor-beta in diabetic nephropathy. Kidney Int 2011; 79: 199-209.

15. Ozbun LL, Martinez A, Jakowlew SB. Differentially expressed nucleolar TGF-beta1 target (DENTT) shows tissue-specific nuclear and cytoplasmic localization and increases TGFbeta1-responsive transcription in primates. Biochim Biophys Acta 2005; 1728: 163-180.

16. Siegel PM, Massague J. Cytostatic and apoptotic actions of TGF-beta in homeostasis and cancer. Nat Rev Cancer 2003; 3: 807-821.

17. Derynck R, Akhurst RJ, Balmain A. TGF-beta signaling in tumor suppression and cancer progression. Nat Genet 2001; 29: 117-129.

18. Grimes JA, Nielsen SJ, Battaglioli E, Miska EA, Speh JC, Berry DL et al. The co-repressor $\mathrm{mSin} 3 \mathrm{~A}$ is a functional component of the REST-CoREST repressor complex. J Biol Chem2000; 275: 9461-9467.

19. Andres ME, Burger C, Peral-Rubio MJ, Battaglioli E, Anderson ME, Grimes J et al. CoREST: a functional corepressor required for regulation of neural-specific gene expression. Proc Natl Acad Sci USA 1999; 96: 9873-9878.

20. Lunyak VV, Burgess R, Prefontaine GG, Nelson C, Sze SH, Chenoweth J et al. Corepressordependent silencing of chromosomal regions encoding neuronal genes. Science 2002; 298: 1747-1752.

21. Zeng W, Kong Q, Li C, Mao B. Xenopus RCOR2 (REST corepressor 2) interacts with ZMYND8, which is involved in neural differentiation. Biochem Biophys Res Commun 2010; 394: 1024-1029.

22. You A, Tong JK, Grozinger CM, Schreiber SL. CoREST is an integral component of the CoREST- human histone deacetylase complex. Proc Natl Acad Sci USA 2001; 98: 1454-1458.

23. Ballas N, Battaglioli E, Atouf F, Andres ME, Chenoweth J, Anderson ME et al. Regulation of neuronal traits by a novel transcriptional complex. Neuron 2001; 31: 353-365.

24. Roopra A, Sharling L, Wood IC, Briggs T, Bachfischer U, Paquette AJ et al. Transcriptional repression by neuron-restrictive silencer factor is mediated via the Sin3-histone deacetylase complex. Mol Cell Biol 2000; 20: 2147-2157.

25. Naruse $\mathrm{Y}$, Aoki T, Kojima T, Mori N. Neural restrictive silencer factor recruits $\mathrm{mSin} 3$ and histone deacetylase complex to repress neuron-specific target genes. Proc Natl Acad Sci USA 1999; 96: 13691-13696.

26. Huang Y, Myers SJ, Dingledine R. Transcriptional repression by REST: recruitment of Sin3A and histone deacetylase to neuronal genes. Nat Neurosci 1999; 2: 867-872.

27. Kortlever RM, Nijwening JH, Bernards R. Transforming growth factor-beta requires its target plasminogen activator inhibitor-1 for cytostatic activity. J Biol Chem2008; 283: 24308-24313.

28. Dennler S, Itoh S, Vivien D, ten Dijke P, Huet S, Gauthier JM. Direct binding of Smad3 and Smad4 to critical TGF beta-inducible elements in the promoter of human plasminogen activator inhibitor-type 1 gene. EMBO J 1998; 17: 3091-3100.

29. Mulligan P, Westbrook TF, Ottinger M, Pavlova N, Chang B, Macia E et al. CDYL bridges REST and histone methyltransferases for gene repression and suppression of cellular transformation. Mol Cell 2008: 32: 718-726.

30. Douma S, Van Laar T, Zevenhoven J, Meuwissen R, Van Garderen E, Peeper DS. Suppression of anoikis and induction of metastasis by the neurotrophic receptor TrkB. Nature 2004; 430: 1034-1039.

31. Li Z, Tognon CE, Godinho FJ, Yasaitis L, Hock H, Herschkowitz Jl et al. ETV6-NTRK3 fusion oncogene initiates breast cancer from committed mammary progenitors via activation of AP1 complex. Cancer Cell 2007; 12: 542-558.

32. Tognon C, Knezevich SR, Huntsman D, Roskelley CD, Melnyk N, Mathers JA et al. Expression of the ETV6-NTRK3 gene fusion as a primary event in human secretory breast carcinoma. Cancer Cell 2002; 2: 367-376. 
33. Knezevich SR, McFadden DE, Tao W, Lim JF, Sorensen PH. A novel ETV6-NTRK3 gene fusion in congenital fibrosarcoma. Nat Genet 1998; 18: 184-187.

34. Eguchi M, Eguchi-Ishimae M, Tojo A, Morishita K, Suzuki K, Sato Y et al. Fusion of ETV6 to neurotrophin-3 receptor TRKC in acute myeloid leukemia with $t(12 ; 15)(p 13 ; q 25)$. Blood 1999; 93: 1355-1363.

35. Jin W, Yun C, Kwak MK, Kim TA, Kim SJ. TrkC binds to the type II TGF-beta receptor to suppress TGF-beta signaling. Oncogene 2007; 26: 7684-7691.

36. Jin W, Kim BC, Tognon C, Lee HJ, Patel S, Lannon CL et al. The ETV6-NTRK3 chimeric tyrosine kinase suppresses TGF-beta signaling by inactivating the TGF-beta type II receptor. Proc Natl Acad Sci USA 2005; 102: 16239-16244.
37. Sometani A, Kataoka H, Nitta A, Fukumitsu H, Nomoto H, Furukawa S. Transforming growth factor-beta1 enhances expression of brain-derived neurotrophic factor and its receptor, TrkB, in neurons cultured from rat cerebral cortex. J Neurosci Res2001; 66: 369-376.

38. Pierce DF Jr., Johnson MD, Matsui Y, Robinson SD, Gold LI, Purchio AF et al. Inhibition of mammary duct development but not alveolar outgrowth during pregnancy in transgenic mice expressing active TGF-beta 1. Genes Dev1993; 7: 2308-2317.

39. Moses H, Barcellos-Hoff MH. TGF-beta biology in mammary development and breast cancer. Cold Spring Harb Perspect Biol2011; 3: a003277.

40. Jin W, Kim GM, Kim MS, Lim MH, Yun C, Jeong J et al. TrkC plays an essential role in breast tumor growth and metastasis. Carcinogenesis 2010; 31: 1939-1947.

Supplementary Information accompanies this paper on Cell Death and Differentiation website (http://www.nature.com/cdd) 